\title{
Energy Efficiency in Wireless Sensor Networks: Selected Papers from IEEE PIMRC 2008
}

\author{
Alain Sibille $\cdot$ Mischa Dohler
}

Published online: 17 July 2009

(C) Springer Science+Business Media, LLC 2009

Wireless Sensor Networks (WSNs) have emerged as perhaps the most important networking paradigm of recent times, both in terms of their commercial potential and also from a scientific point of view. WSNs are important commercially because of their many applications, spanning from civil to military applications such as structural monitoring of bridges, monitoring of wildlife, tracking of contaminants in the soil and atmosphere. Some of these applications have already proved to be commercially viable and indeed financially very attractive. WSNs are also very important from a scientific point of view, because of their unique features with respect to other types of wireless networks: nodes are typically immobile, and are required to carry a specific type of traffic (which is advantageous), have extreme restrictions on the energy they consume, the processing power they have, and the antennas they can use (which is disadvantageous); in addition, the data created at

Wireless Sensor Networks (WSNs) have emerged as perhaps the most important networking paradigm of recent times, both in terms of their commercial potential and also from a scientific point of view. The papers that appear in this issue have been carefully selected from the best IEEE PIMRC 2008 conference papers which addressed the issue of energy efficiency in WSNs. The final set of papers addresses issues at physical, medium access control, networking and to some extend application layers. All of these papers take into account the unique peculiarities, advantages, and shortcomings of wireless sensor networks, and propose algorithms and analyses that significantly advance research in the field. Note that this activity has been partly supported by the NoE-216715 NEWCOM++.
A. Sibille
ENSTA-ParisTech, Paris, France
e-mail: alain.sibille@ensta.fr
M. Dohler $(\bowtie)$
CTTC, Barcelona, Spain
e-mail: mischa.dohler@cttc.es

different sensors may be strongly correlated, which can be beneficial but requires only good algorithms that take advantage of it. Therefore, in the last few years we have experienced an important shift in research activities, moving from general purpose wireless networks to more focused topics specifically on WSNs, taking into account their specific advantages and peculiarities. An important recurring theme in research in this field is the need for energy efficiency, which is the thread of this special issue.

The papers which appear in this issue have been carefully selected from the best IEEE PIMRC 2008 conference papers addressing the issue of energy efficiency in WSNs. The respective authors have been asked to provide a significantly extended version of the conference paper, which has subsequently undergone a rigorous review process according to the IJWIN publication standards prior to acceptance. The final set of papers addresses issues at physical, medium access control and networking layers, and to some extent at the application layer. All of these papers take into account the unique peculiarities, advantages, and shortcomings of wireless sensor networks, and propose algorithms and analyses that significantly advance research in the field. We sincerely hope you will enjoy reading them as much as we did.

The author of the first paper "Power Management and Data Rate Maximization in Wireless Energy Harvesting Sensors" by Chandra R. Murthy, deals with the problem of power management and throughput maximization for energy neutral operation when using increasingly important but so far rarely analyzed Energy Harvesting Sensors (EHS) to send data over wireless links. The EHS is assumed to be able to harvest energy at a constant rate, and use a fixed part of the energy harvested in a slot for measuring the channel state. The rest of the harvested energy is available for transmission, but it can also be stored in an 
inefficient battery if not fully utilized. A framework is developed under which the system designer can optimize several parameters of the EHS which determine the average data rate performance when the channel is obeying Rayleigh fading.

The second paper, "Radio-Triggered Wake-ups with Addressing Capabilities for Extremely Low Power Sensor Network Applications" by Junaid Ansari, Dmitry Pankin and Petri Mähönen, proposes to use a radio triggered wake-up with built-in addressing capabilities (RTWAC), which allows lowering the energy consumption of WSNs even further by suppressing the idle duration of current consumption. The approach relies on an external low-cost hardware wake-up circuit attached to the microcontroller of a sensor node, which is able to process a special kind of out-of-band modulated wake-up signal. The novelty here is that the modulated signal contains data enabling to distinguish between differently addressed nodes, in order to avoid undesired node wake-ups.

A step towards practically viable WSN roll-outs has been taken in the third paper, "Energy Efficient Reliable Data Collection in Wireless Sensor Networks with Asymmetric Links" by Ren P. Liu, Zvi Rosberg, Iain B. Collings, Carol Wilson, Alex Y. Dong and Sanjay Jha, which capitalizes on the insights obtained from field measurements revealing that radio link asymmetry has a severe impact on reliable data delivery. They analyze the energy efficiency of selected reliability schemes for asymmetric radio links using theoretical models and provide guidelines for retransmission control so as to balance between reliability and energy consumption. They also design two enhancements to the "implicit" ARQ scheme as well as a novel energy efficient and reliable data collection mechanism, addressing the negative effects of asymmetric radio links.

The fourth paper, "Energy-aware Georouting with Guaranteed Delivery in Wireless Sensor Networks with Obstacles" by Essia Hamouda, Nathalie Mitton, Bogdan Pavkovic and David Simplot-Ryl, proposes a novel end-toend localized routing protocol for WSNs which is energyefficient and guarantees delivery. In order to forward a packet, a node $s$ in graph $G$ computes the cost of the energy weighted shortest path between $s$ and each of its neighbors in the forward direction, towards the destination which minimizes the ratio of the cost of the shortest path to the progress (reduction in distance towards the destination). Then it sends the message to the first node on the shortest path and in order to recover from possible local minima traps, the proposed algorithm invokes an energy-aware Face routing which guarantees delivery. This paper is essentially the first to optimize energy consumption of used Face routing.
The fifth paper, "An Energy-Efficient Routing Protocol for Event-Driven Dense Wireless Sensor Networks" by Liuguo Yin, Changmian Wang and Geir E. Oien, proposes a novel minimum cost routing with optimized data fusion protocol. Referred to as MICRO, it is an energy-efficient routing protocol for event-driven dense WSNs and significantly improves on LEACH and PEGASIS. In the routing computation, the proposed scheme exploits a new cost function for energy balancing among sensor nodes, and uses an iterative scheme with optimized data fusion to compute the minimum-cost route for each event-detecting sensor node.

The sixth paper, "Efficient Access Control for Wireless Sensor Data" by Alessandro Sorniotti, Refik Molva, Laurent Gomez, Christophe Trefois, Annett Laube and Piervito Scaglioso, deals with an important but so-far neglected topic of access control to e.g. databases and filesystems in WSNs, which is particularly pertinent in healthcare applications with sensitive data. Enforcing access control in WSNs is a particularly difficult task due to the limited computational capacity of wireless sensor nodes. This paper, however, presents a full-fledged access control scheme for wireless sensor data. Access control is enforced through data encryption, thus embedding access control into the sensor data units. A lightweight key generation mechanism based on cryptographic hash functions is also proposed which allows for hierarchical key derivation. The derived protocol relies on simple operations only, does not require interactions between nodes and data consumers and has minimal storage requirements.

The seventh and final paper, "Modeling and designing efficient data aggregation in WSNs under entropy and energy bounds" by Laura Galluccio, Sergio Palazzo and Andrew T. Campbell, capitalizes on the observation that propagating redundant and highly correlated data through the WSN is costly in terms of system performance and results in energy depletion, network overloading, and congestion. In this paper, they derive a number of important insights concerning the remedy of data aggregation, which have not been discussed in the literature so far. They first estimate the conditions under which aggregation is a costly process in comparison to a non aggregation approach, by considering a realistic scenario where the processing costs related to aggregation of data are not neglected. An underlying assumption is that aggregation should preserve the integrity of data, implying that the entropy of the correlated data sent by sources can be taken into account in order to both decrease the amount of redundant data forwarded to the sink and to perform an overall lossless process. The presented framework serves to investigate the tradeoff between the increase in data aggregation required to 
reduce energy consumption, and the need to maximize information integrity whilst also understanding how aggregation impacts the network propagation delay of a data packet.

We would like to thank the authors of all papers for considering our special issue for disseminating their work and for having significantly worked on the papers to extend them from the respective conference versions. We extend our gratitude to the many, very conscientious reviewers, for sacrificing so much of their time in order to make this special issue a success. Last, but not least, we would like to thank the devoted staff of Springer for their high level of professionalism.

\section{Author Biographies}

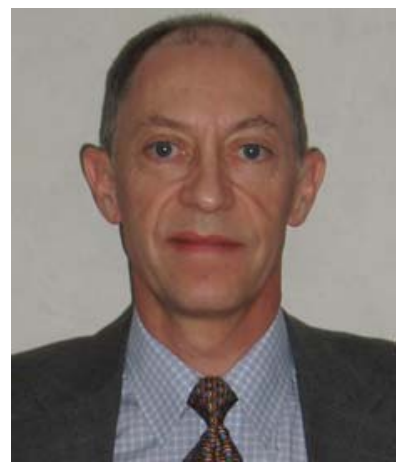

Alain Sibille graduated from Ecole Polytechnique (1977) and from Telecom-ParisTech (1979) in France and obtained the $\mathrm{PhD} /$ habilitation degree in 1985 . He first conducted basic research within France Telecom R\&D and later as a part time independent consultant, then moved to ENSTA-ParisTech where he now leads the Electronics and Computer Engineering department. His current scientific interests lie in ultra wide band communications and systems, and in the interactions between antennas, channels, and signal processing. Prof. Alain Sibille has chaired the European Wireless Conference 2007 in Paris and cochaired the TPC of PIMRC 2008. He was a National delegate in COST 273 European cooperation action "Towards Wireless Broadband Multimedia Networks" and is currently Chair of WG2 "Radio Channel" within the ongoing COST 2100 European Action "Pervasive Mobile \& Ambient Wireless Communications". He is also involved in URSI-France as Vice Secretary General and Vice Chair of Commission C "Signals and Systems".

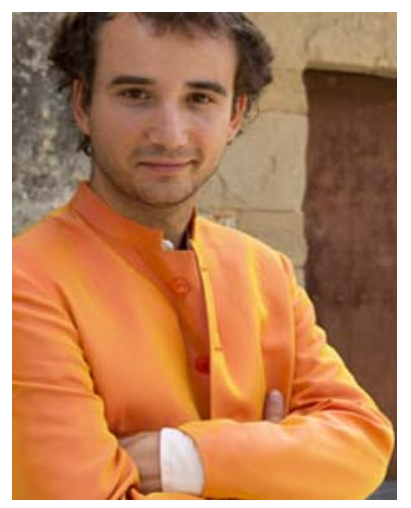

Mischa Dohler is now Senior Researcher with CTTC in Barcelona. Prior to this, from June 2005 to February 2008, he has been Senior Research Expert in the $R \& D$ division of France Telecom working on cooperative communication systems, cognitive radios and wireless sensor networks. From September 2003 to June 2005, he has been lecturer at King's College London, Centre for Telecommunications Research. At that time, he has also been London Technology Network Business Fellow for King's College London, as well as Student Representative of the IEEE UKRI Section and member of the Student Activity Committee of IEEE Region 8 (Europe, Africa, Middle-East and Russia). He obtained his PhD in Telecommunications from King's College London, UK, in 2003, his Diploma in Electrical Engineering from Dresden University of Technology, Germany, in 2000, and his MSc degree in Telecommunications from King's College London, UK, in 1999. Prior to Telecommunications, he studied Physics in Moscow. He has won various competitions in Mathematics and Physics, and participated in the 3rd round of the International Physics Olympics for Germany. In the framework of the Mobile VCE, he has pioneered research on distributed cooperative space-time encoded communication systems, dating back to December 1999. He has published more than 110 technical journal and conference papers at a citation h-index of 17 and citation g-index of 34, holds several patents, co-edited and contributed to several books, has given numerous international short-courses, and participated in standardisation activities. He has been TPC member and co-chair of various conferences, such as technical chair of IEEE PIMRC 2008 held in Cannes, France. He is editor for numerous IEEE and non-IEEE journals and a Senior Member of the IEEE. 